\title{
Value in cardiac surgery: The price of saving
}

\author{
Robbin G. Cohen, MD, MMM
}

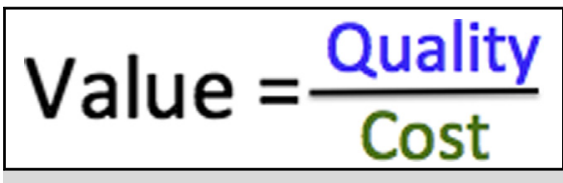

Medicare is the customer in the cardiac surgery value equation, patients the benefactors.

\section{Central Message}

Clinical and financial strategies that satisfy the demands of the Affordable Care Act to provide cost-effective care must result in a value equation that benefits patients.

See Article page 190.
The concept of value in health care, where
value $=$ quality $/$ cost, has been circulating for decades. In 2010, Porter ${ }^{1}$ declared that value was neither an "abstract ideal nor a code word for cost reduction," and that it should define the framework for performance improvement in health care. He also stressed that value should always be defined around the customer, and in a well-functioning health care system the creation of value for patients should determine the rewards for all other actors in the system.

The quest for value poses several challenges to the cardiac surgeon. How do you decrease hospital stay and reduce skilled nursing facility and rehabilitation center use without increasing readmissions? How do you work within new payer mandates without annihilating your group and hospital margins from increased internal resource acquisition and utilization? What changes in clinical care protocols have the greatest chance of actually decreasing costs, and how should these cost savings be measured?

In their article in this issue of the Journal, Koeckert and colleagues $^{1}$ at New York University have done a nice job describing how they successfully "reengineered" their postdischarge management for patients undergoing cardiac valve surgery or transcatheter valve replacements to adapt to the Payment Model 2 of the Bundled Payments for Care Improvement initiative set out by the Center for Medicare and Medicaid Innovation (CMMI) in 2010.2 Although it seems offensive that the customer influencing these changes is the CMMI and not the patients themselves, the end result has the potential to be positive for all stakeholders.

For the New York University group, ${ }^{2}$ there were lowhanging fruit on which to begin (an initial 70\% discharge rate to inpatient rehabilitation or skilled nursing facilities), and they demonstrated significant improvements in 30-day readmission rates by applying a combination of common currently used tactics. These included better attention to preoperative risk assessment, improved patient education with identification of discharge goals, identification of common readmission etiologies (ie, volume overload and atrial fibrillation), and increased postdischarge surveillance by both physicians and ancillary care personnel (cardiac nurse practitioners). They also used a "less than 48-hour hospital observation" system in lieu of actual hospital readmission, which some might consider an attempt at gaming the system. One strategy that they did not consider was aggressive negotiations regarding the prices of valves and other equipment, which can save thousands in cost on every case.

It is difficult to determine which of these strategies contributed the most to cost savings, as well as how much Koeckert and colleagues ${ }^{1}$ or their institution had to spend to improve their results. For instance, patient education must be offered early in the process, be repetitive, and take place in multiple settings by multiple providers to be effective. ${ }^{3}$ The price of adding ancillary care members to the cardiac surgical team to enhance education and postdischarge patient surveillance can't be cheap. Regardless, the CMMI has made it clear that their influence is here to stay, that some providers will be rewarded and others punished, and that despite decades of trying we still have a lot to learn when it comes to value in cardiac surgery. If in the long run the patients are the ultimate benefactors of the value equation, maybe it's not such a painful idea after all.

\section{References}

1. Porter ME. What is value in health care? N Engl J Med. 2010;363:2477-81.

2. Koeckert MS, Ursomanno PA, Williams MR, Querijero M, Zias EA, Loulmet DF, et al. Reengineering valve patient's postdischarge management for adapting to bundled payment models. J Thorac Cardiovasc Surg. 2017;154:190-8.

3. Ronco M, Iona L, Fabbro C, Bulfone G, Palese A. Patient education outcomes in surgery: a systematic review from 2004 to 2010. Int J Evid Based Healthc. 2012; 10:309-23. 